\title{
Cognição organizacional: revisão, conceitualização e contexto estratégico
}

\author{
Farley Nobre ${ }^{a *}$, Andrew Tobias ${ }^{\mathrm{b}}$, David Walker ${ }^{\mathrm{b}}$ \\ aUniversidade Federal do Paraná, Curitiba, PR, Brasil \\ bUniversity of Birmingham, Birmingham WM, United Kingdom \\ *fsmnobre@gmail.com
}

\begin{abstract}
Resumo
Pesquisas sobre cognição nas organizações têm sido fundamentadas em áreas e estudos multidisciplinares que se desenvolveram principalmente nos últimos cinquenta anos. Cognição organizacional tem recebido contribuições de abordagens computacionais e interpretativas e pode ser compreendida como uma área de teoria das organizações. Todavia, apesar de apoiar esses desenvolvimentos, este artigo aponta para a necessidade de se avançar e propor definições mais concisas e precisas sobre cognição organizacional, na direção de conceitualizações que possam esclarecer a sua distinção e suas fronteiras com os conceitos de inteligência, autonomia, aprendizagem e gestão do conhecimento organizacional. Adicionalmente, este artigo destaca a necessidade de concentração de maiores esforços para a redução da distância entre teoria e prática no campo da cognição organizacional. Para atender a essas necessidades, este trabalho contribui para o campo da cognição organizacional através de revisão de literatura, conceitualizações e a sua introdução no contexto da administração estratégica de recursos.

Palavras-chave: Cognição organizacional. Habilidades organizacionais. Recursos estratégicos. Incerteza ambiental.
\end{abstract}

\section{Introdução}

Estudos sobre cognição nas organizações tiveram início aproximadamente nos anos $1950 \mathrm{em}$ um ambiente de pesquisa extensiva e multidisciplinar influenciada pelos desenvolvimentos em teoria geral dos sistemas, psicologia cognitiva e social, inteligência artificial e ciências cognitivas, psicologia social nas organizações, sociologia do conhecimento, aprendizagem organizacional e gestão do conhecimento. Recentemente, a área de estudos da cognição nas organizações foi denominada “cognição organizacional”, sendo assim abordada nas publicações de Eden \& Spender (1998), Hodgkinson \& Healey (2008), landoli \& Zollo (2007), Lant \& Shapira (2001), Nicolini (1999), Nobre et al. (2009a, b; 2014), Nobre \& Walker (2011) e Walsh (1995).

Pesquisas sobre cognição nas organizações têm suas raízes nas publicações de Simon (1947), sobre comportamento administrativo, e March \& Simon (1958) sobre organizações. Nestas publicações, a organização está associada a sistemas cognitivos cuja estrutura comporta uma rede de agentes e processos os quais são organizados através de relações verticais e horizontais. Sob esta perspectiva, a organização beneficia indivíduos e grupos através da extensão das suas limitações cognitivas para modelos mais avançados de racionalidade (Simon, 1997a, b). Contudo, o significado desta perspectiva foi desenvolvido de forma distinta por alguns pesquisadores que a dividiram em dois polos principais denominados de abordagens computacionais e interpretativas (Lant \& Shapira, 2001). A abordagem computacional investiga os processos através dos quais a organização processa a informação e, portanto, associa a organização a máquinas ou mecanismos de processamento de informação. Sua ênfase está sobre informação e eficiência. Esta abordagem está fundamentada na psicologia cognitiva, ciências cognitivas e inteligência artificial. Alguns trabalhos que influenciaram a abordagem computacional incluem as perspectivas cognitivas e de processamento de informação propostas em Human Problem Solving por Newell \& Simon (1972), Unified Theories of Cognition por Newell (1990) e The Society of Mind por Minsky (1986), além dos estudos sobre processos 
de tomada de decisão, comportamento e economia de firmas como introduzidos em $A$ Behavioral Theory of the Firm por Cyert \& March (1963). Mais recente, a abordagem computacional foi estendida para uma perspectiva mais avançada de computação distribuída, tendo recebido maiores contribuições do campo de Teoria Computacional de Organizações, o qual tem sido largamente desenvolvido na Universidade de Carnegie Mellon (Carley \& Gasser, 1999; Prietula et al., 1998). No contexto dos estudos organizacionais, a abordagem computacional oferece recursos para a análise de capacidade, desempenho e eficiência do processamento de informação de distintos modelos de organizações. Esta perspectiva do processamento de informação organizacional foi intensivamente desenvolvida por Galbraith (1977) e sua aplicação foi ilustrada por Premkumar et al. (2005).

A abordagem interpretativa examina o processo de criação de significado para a informação em um contexto social e está relacionada aos sistemas de conhecimento e às coletividades sociais. Sua ênfase está sobre conhecimento e coletividades. Esta abordagem está fundamentada na sociologia do conhecimento (Berger \& Luckmann, 1967; Gurvitch, 1971; Holzner \& Marx, 1979; Stehr \& Meja, 2005), na psicologia social das organizações (Katz \& Kahn, 1967; Weick, 1979, 1995), na cognição social (Bless et al., 2004; Brewer \& Hewstone, 2004), e mais recentemente na gestão do conhecimento (Alavi \& Leidner, 2001; Harvard Business Review, 1998; Ichijo \& Nonaka, 2006; Nonaka, 2005) e na aprendizagem organizacional (Argyris, 1999; Dierkes et al., 2003), onde este último tópico também tem sido relacionado à criação, armazenagem e transferência do conhecimento nas organizações (Argote, 2007).

Alguns autores (landoli \& Zollo, 2007) têm classificado estas duas abordagens da cognição organizacional de acordo com a dicotomia entre o hard, que significa uma perspectiva de processamento de informação objetiva e quantitativa relacionada à abordagem computacional, e o soft que representa uma perspectiva de sense making, ou seja, de interpretação subjetiva e qualitativa da informação para criação de significado e conhecimento, sendo esta associada à abordagem interpretativa. No contexto das escolas de teoria das organizações, este artigo propõe que estes dois importantes fluxos de pesquisas em cognição organizacional podem ser associados a dois extremos que delimitam um espectro contínuo de modelos organizacionais que envolvem os conceitos de sistemas abertos racionais e naturais (Scott, 1998, p. 31-120). Sobre um extremo está a abordagem computacional, a qual pode ser associada aos modelos organizacionais de sistemas abertos racionais. Sobre outro extremo está a abordagem interpretativa, a qual pode ser associada aos modelos organizacionais de sistemas abertos naturais. Os sistemas racionais superestimam a estrutura normativa, a especificidade de objetivos, a formalização, o projeto e desempenho organizacional, e por isso se assemelham às características das organizações mecanicistas. Por outro lado, os sistemas naturais superestimam a estrutura comportamental, a complexidade de objetivos múltiplos, a adaptação e sobrevivência da organização, e por isso têm maiores similaridades às organizações orgânicas (Scott, 1998). A Tabela 1 apresenta um resumo das principais características destes dois extremos.

Se esses extremos ajudam ou não os pesquisadores a classificarem seus paradigmas, pode-se afirmar que a maioria das perspectivas sobre cognição organizacional está situada em algum lugar no contínuo entre as abordagens computacionais e interpretativas. Adicionalmente, pesquisas mais recentes têm revelado que a integração destas duas abordagens é o melhor caminho para o progresso neste campo de estudos (Choo, 2005; Lant \& Shapira, 2001).

\section{Proposta, motivação e metodologia}

Este artigo contribui para o campo da cognição organizacional através de revisão de literatura, conceitualizações e a sua inserção no contexto da administração de recursos estratégicos.

Em sua proposta, este trabalho se baseia na premissa de que pesquisas em cognição organizacional precisam ampliar seus esforços no domínio da

Tabela 1. Características extremas das abordagens computacionais e interpretativas.

\begin{tabular}{|c|c|c|}
\hline Características & Abordagem computacional & Abordagem interpretativa \\
\hline Fundamentada em: & $\begin{array}{l}\text { Psicologia cognitiva, ciências cognitivas, inteligência } \\
\text { artificial }\end{array}$ & $\begin{array}{l}\text { Sociologia do conhecimento, psicologia social } \\
\text { das organizações, cognição social, gestão do } \\
\text { conhecimento, aprendizagem organizacional }\end{array}$ \\
\hline Associada a: & Máquinas de processamento de informação & Sistemas do conhecimento, coletividades sociais \\
\hline Foco em: & Informação e eficiência & Conhecimento, coletividades \\
\hline Dicotomia entre: & $\begin{array}{l}\text { Hard, processamento de informação objetiva e } \\
\text { quantitativa }\end{array}$ & $\begin{array}{l}\text { Soft, interpretação subjetiva e qualitativa, sense } \\
\text { making }\end{array}$ \\
\hline Perspectivas organizacionais: & Sistemas abertos racionais, modelos mecanicistas & Sistemas abertos naturais, modelos orgânicos \\
\hline
\end{tabular}


conceitualização. A necessidade de melhoria de conceitualização, ou de definição de conceitos sobre cognição organizacional, pode ser justificada pelo fato de que este campo de estudos tem sido fragmentado em áreas diversas, e, por conseguinte, sobreposto de forma obscura a outros conceitos que envolvem aprendizagem organizacional e gestão do conhecimento. Com o propósito de contribuir para esse domínio, este artigo estende a literatura propondo princípios e definições que delineiam conceitualizações sobre cognição organizacional. Em sua proposta, este trabalho compreende cognição como a principal habilidade que pode proporcionar inteligência, autonomia, aprendizagem e gestão do conhecimento aos indivíduos, grupos e organizações. Portanto, este artigo propõe e define cognição como o principal elemento entre as habilidades organizacionais. Ainda sob essa perspectiva, este trabalho caracteriza distinções entre cognição organizacional e conceitos de inteligência, autonomia, aprendizagem e gestão do conhecimento organizacional. Relações e fronteiras entre esses conceitos são apresentadas, e, apesar de serem distintos, eles formam habilidades complementares na organização. Tais conceitos, quando agrupados, formam um conjunto de habilidades na organização. A partir da revisão e proposta de conceitualizações, este artigo sugere a formação de uma base para estudos de uma teoria de habilidades organizacionais a qual é proposta no contexto da administração estratégica de recursos.

Prosseguindo a partir das suas conceitualizações, este artigo propõe definições de Grau Relativo de Cognição Organizacional e Nível Relativo de Incerteza Ambiental, onde estas definições apresentam cognição como principal habilidade estratégica na organização para o efetivo controle, governo e redução do nível de incerteza no ambiente. Fundamentando-se nas suas propostas, este trabalho se concentra em estratégias de organizações que priorizam a busca de altos graus de cognição organizacional para a administração de altos níveis de incerteza ambiental, e, adicionalmente, define cognição como uma habilidade estratégica de prioridade para o desenvolvimento da vantagem competitiva da organização.

0 desenvolvimento desta pesquisa está fundamentado em processos de teorização (Bailey, 1982) e inclui as seguintes etapas: (1) definição do problema, o qual está relacionado à necessidade de melhoria na conceitualização de cognição organizacional, (2) definição das principais variáveis e suas fronteiras, as quais incluem cognição organizacional e incerteza ambiental, (3) definição do escopo da classe de organizações considerada neste artigo, (4) definição das habilidades estratégicas organizacionais e proposta de um modelo dinâmico de criação de vantagem competitiva, (5) definição das relações e causalidades entre as principais variáveis de estudo (cognição organizacional e incerteza ambiental), análise da influência de uma variável sobre a outra e esboço de implicações teóricas e práticas, e (6) apresentação das contribuições e conclusões finais.

\section{Variáveis: incerteza e cognição}

Esta seção apresenta as variáveis de estado e de controle (variáveis de estado descrevem a posição, condição, trajetória ou comportamento de um sistema. Variáveis de controle influenciam variáveis de estado e são utilizadas para regular ou governar o estado ou comportamento de um sistema) que são do interesse deste artigo. Incerteza no ambiente é definida como variável de estado e cognição na organização é definida como variável de controle. Isso sugere que incerteza pode ser controlada ou regulada, e, em parte, reduzida, pelas habilidades cognitivas na organização.

\subsection{Incerteza e complexidade ambiental}

A incerteza ambiental pode ser compreendida como o nível de incerteza associado ao ambiente da organização, ou, ainda, como o nível de incerteza que os participantes e grupos da organização percebem seus ambientes (Ducan, 1972). Adicionalmente, no campo das teorias da contingência, alguns autores têm definido a incerteza como uma variável ou categoria que condiciona a organização como dependente do seu ambiente (Galbraith, 1977). Por conseguinte, o projeto organizacional (Galbraith, 2002) e a escolha dos elementos da organização (Scott, 1998) dependem do conceito de incerteza cujo significado pode ser associado a proposições da racionalidade limitada (Simon, 1982a, b; 1997a, b). Sob essa perspectiva, incerteza está associada à falta de informação e insuficiência de cognição para o processamento de informações e interpretação (Nobre et al., 2009a, b, c). De maneira complementar, a complexidade do ambiente é contingente ao nível de incerteza ambiental compreendido pela organização e a complexidade do ambiente de tarefas é contingente ao nível de incerteza compreendido pelos participantes da organização. Portanto, pode-se deduzir que quanto maior o nível de incerteza ambiental, maior é o nível de complexidade ambiental que a organização compreende e necessita administrar (Nobre \& Walker, 2011).

De acordo com o escopo proposto, incerteza pode ser caracterizada por incompletude, vagueza, confusão e dissonância de informação. Resumidamente, incompletude representa a falta de partes relevantes 
e de conteúdo na informação, podendo ser causada por fatores técnicos, econômicos, políticos e sociais. Vagueza está relacionada à nebulosidade (ensaios sobre vagueza foram apresentados por Black (1937, 1963), e teorias sobre lógica nebulosa foram propostas por Zadeh (1973). Nestas teorias, categorias nebulosas são definidas por classes de objetos que não possuem fronteiras bem definidas, e, portanto, a transição entre pertinência e não pertinência dos seus elementos acontece de forma gradual e não abrupta, Klir \& Folger, 1988), e, portanto, representa o tipo de incerteza encontrado com maior frequência nos conceitos formados e manipulados pela mente dos participantes nas organizações (Nobre et al., 2009a). De acordo com a psicologia cognitiva, estes conceitos são denominados conceitos naturais (Conceitos naturais são representações mentais de classes de objetos físicos e abstratos que possuem propriedades comuns como os atributos de cor [vermelho, amarelo, verde], tamanhos [pequeno, médio, grande], velocidade [devagar, rápido], etc. Conceitos naturais são inerentemente caracterizados por fronteiras nebulosas uma vez que alguns de seus membros são melhores exemplos da categoria que outros, Bernstein et al., 2008; Lefrançoies, 1995; Zadeh, 1999) e são caracterizados por fronteiras nebulosas (Bernstein et al., 2008; Eysenck \& Keane, 2005). Confusão é um tipo de ambiguidade que reside na dificuldade de escolha de uma entre várias alternativas. E dissonância representa outra espécie de ambiguidade que se refere às diferenças de significado existentes entre duas ou mais informações. De acordo com estas definições, observa-se que incompletude, vagueza e confusão de informação apresentam grandes similaridades aos respectivos conceitos de inaceitabilidade (do original unacceptability), incerteza e incomparabilidade que são apresentados em March \& Simon (1958), e, os quais, representam fontes de conflitos intraindividuais em processos de decisão. Adicionalmente, dissonância de informação apresenta similaridade às fontes de conflitos de grupo, as quais incluem diferenças nas percepções, valores, culturas e motivações dos participantes nas organizações. Ressalta-se que os conceitos de conflitos intraindividuais e de grupo nas organizações foram propostos por March \& Simon (1958), e, mais recentemente, foram analisados em Nobre et al. (2009a, c).

\subsection{Cognição na organização}

Cognição na organização ou cognição organizacional abrange as perspectivas de processos e representações nas organizações. Por um lado, quando associada a processos, cognição envolve sentido, percepção, atenção, identificação e formação de conceitos, categorização, representação e organização do conhecimento, memória, linguagem, decisão e resolução de problemas (Nobre et al., 2009a; 2014). Estes processos cognitivos exercem a função de mediadores de efeitos de eventos externos ou estímulos sobre as decisões, comportamentos e ações de indivíduos, grupos e organizações, em resposta às suas experiências. A organização capaz de integrar estes processos é denominada organização cognitiva (Nobre et al., 2009a), e, em particular, a organização que integra processos de interpretação (ou sense making), criação do conhecimento, e tomada de decisão é descrita como organização do conhecimento (Choo, 2005). Nestas classes de organizações, a informação no ambiente é sensibilizada e interpretada, e seu significado é socialmente elaborado e construído na mente dos indivíduos da organização. Conhecimento tácito é convertido em conhecimento explícito, e vice-versa, e o conhecimento individual é expandido para níveis ontológicos mais elevados, de grupo, organizacional e interorganizacional, de acordo com os processos do ciclo espiral de criação do conhecimento (lchijo \& Nonaka, 2006). Os processos de sensibilização e interpretação (ou sense making), e de criação do conhecimento, proporcionam à organização ambiente e contextos apropriados para tomada de decisões, resolução de problemas, aprendizagem e atuações. Por outro lado, quando associada a representações, cognição se torna sinônimo de imagens mentais, modelos de conhecimento e mapas cognitivos elaborados e construídos a partir de experiências e aprendizagem de indivíduos, grupos e organizações. Representações exercem a função-chave de direcionar comportamentos na ausência de estímulos do ambiente (Brewer \& Hewstone, 2004).

\subsubsection{Grau de cognição organizacional}

Cognição organizacional pode também ser associada a graus de cognição na organização (Nobre et al., 2009a, b). Sob um olhar, o grau de cognição organizacional pode ser relacionado ao nível de elaboração ou complexidade com que indivíduos, grupos e organizações percebem seus ambientes e formam seus mapas cognitivos. Nesse caso, o grau de complexidade cognitivo pode ser associado à quantidade de construtos horizontais e ao número de niveis verticais em profundidade que integram o mapa cognitivo (Calori et al., 1994). Sob outro olhar, grau de cognição pode ser simbolicamente associado a medidas tangíveis e intangíveis de processos e representações. Em Nobre et al. (2009a, 2014), por exemplo, os autores apresentam um estudo de caso sobre uma empresa multinacional que atua no ramo de telecomunicações e desenvolvimento de software, 
onde, neste estudo, o grau de cognição organizacional foi associado a níveis de maturidade, capacidade e desempenho de processos da organização, e também foi correlacionado a resultados de aprendizagem organizacional. Análises qualitativas e quantitativas do estudo de caso indicaram que melhorias nos níveis de maturidade e desempenho de processos da organização estavam associadas a melhorias nos níveis de cognição organizacional; e também mostraram que melhorias na aprendizagem organizacional estavam relacionadas a melhorias na cognição organizacional. Ainda neste estudo, observou-se que a melhoria no grau de cognição proporcionou uma redução no nível de incerteza do ambiente técnico da organização, onde neste caso a incerteza foi associada à complexidade dos ambientes de tarefa de desenvolvimento de projetos de software. Métodos similares haviam sido adotados por outros autores que associaram ganhos de produtividade e desempenho a práticas de aprendizagem organizacional (Argote, 2007). Noutro trabalho, que investiga a relação entre grau de cognição e nível de sustentabilidade em empresas listadas no Índice de Sustentabilidade Empresarial (ISE) da Bolsa de Valores de São Paulo, os autores (Nobre \& Ribeiro, 2013) operacionalizaram o conceito de grau de cognição, na forma matemática, representando-o por uma medida relacionada à diferenciação e à integração de mapa cognitivo, em que a Diferenciação (D) equivale ao número de construtos (ou categorias), e a Integração (1), ao número de links (ou relações entre as categorias e subcategorias) presentes em um mapa cognitivo. Portanto, pode-se afirmar que a operacionalização do conceito de cognição ou grau de cognição depende do contexto da sua aplicação.

Uma vez definidas as variáveis de interesse deste artigo, a próxima seção apresenta o escopo da organização.

\section{Escopo da organização}

A classe de organizações considerada neste artigo atende aos critérios e características dos Sistemas Centrados no Cliente (Nobre, 2011; Nobre et al., 2009a, b). Os principais elementos nestas organizações são estrutura social, objetivos, tecnologias e participantes (Scott, 1998, p. 17-23). Estas organizações são orientadas por estratégias que contribuem para 0 cumprimento, ou satisfice (Simon, 1997b) de objetivos. Adicionalmente, elas interagem com o ambiente e são produtoras de bens e serviços. Em organizações deste tipo, busca-se de forma intensiva um algo grau de cognição organizacional, e, consequentemente, procura-se atingir altos níveis de flexibilidade e agilidade organizacional, proporcionando à empresa capacidade de administrar altos níveis de incerteza ambiental e de operar através de customização em massa (Pine, 1999), além de oferecer um ambiente de imersividade (Imersividade (tradução do original immersiveness) representa a habilidade de um sistema organizacional em interagir com clientes ou consumidores, sejam humanos ou máquinas cognitivas, de uma forma amigável, proporcionando a imersão deles na organização através de técnicas de realidade virtual, simulações ou operações reais, Nobre et al., 2009a, b) aos seus clientes e consumidores (Nobre et al., 2009a, b). Nestas organizações, máquinas cognitivas (Máquinas cognitivas podem ser definidas como classes particulares e avançadas de sistemas de gestão do conhecimento. Estudos amplos sobre projeto e análise de máquinas cognitivas nas organizações são propostos em Nobre, 2011; Nobre et al., 2009a, b, c) são agentes promissores e fundamentais para governança, automação e controle de toda a empresa. Além disso, organizações deste tipo podem ser projetadas através dos conceitos de Redes de Gestão de Organizações Computacionais (Redes de Gestão de Organizações Computacionais (ou Computational Organization Management Networks - COMN) deverão exercer um papel fundamental no desenvolvimento de futuras organizações onde máquinas cognitivas serão os principais agentes de governança, automação e controle da empresa como um todo (Nobre, 2011; Nobre et al., 2009a, b).

A próxima seção apresenta conceitos fundamentais de habilidades organizacionais e enfatiza princípios sobre cognição organizacional e suas fronteiras com inteligência, autonomia, aprendizagem e gestão do conhecimento organizacional.

\section{Habilidades estratégicas na organização}

\subsection{Contexto e definições preliminares}

\subsubsection{Recursos estratégicos}

Recursos estratégicos podem ser entendidos como elementos principais utilizados nos processos de produção da empresa, subdividindo-se em recursos tangíveis e intangíveis (Takahashi \& Takahashi, 2007). Este artigo apoia esta definição ao assumir a perspectiva de que recursos estratégicos podem ser considerados como o conjunto de elementos internos à organização, envolvendo estrutura social, objetivos, tecnologias, participantes e ambientes de tarefas (Scott, 1998, p. 17-22), e, além disso, assume que estes recursos se aplicam aos níveis técnicos ou de produção, administrativo e institucional nas organizações. Nesta definição, a organização interage com o ambiente externo para aquisição, processamento, criação e gestão de novos recursos estratégicos. 


\subsubsection{Habilidades organizacionais}

Habilidade é algo que está relacionado à capacidade de agir mentalmente, fisicamente, financeiramente, legalmente, juridicamente, institucionalmente, entre outras formas. Habilidades cognitivas se referem especificamente a capacidades mentais (Ree et al., 2002). No contexto deste artigo, habilidade cognitiva envolve processos e representações que podem proporcionar inteligência, autonomia, aprendizagem e gestão do conhecimento aos indivíduos, grupos e organizações. Portanto, este artigo define que cognição é a principal habilidade organizacional. Sob esta perspectiva, as habilidades na organização, ou, simplesmente, habilidades organizacionais, abrangem conceitos de cognição, inteligência, autonomia, aprendizagem e gestão do conhecimento. Estas habilidades organizacionais têm um papel fundamental na administração dos recursos estratégicos da organização e elas representam fontes do desenvolvimento de competências essenciais na organização, onde esta perspectiva é inspirada nos contextos estratégicos de teoria da empresa baseada em recursos. Sob esse domínio, este trabalho sugere que este conjunto de habilidades na organização apresenta conotação similar ao contexto de recursos em uma teoria da empresa baseada em recursos (Wernerfelt, 1984), e também ao contexto do conhecimento em uma teoria da empresa baseada no conhecimento (Grant, 1996; Oliveira, 1999; Spender, 1996a, b). Adicionalmente, quando tratadas sob a perspectiva de processos dinâmicos e de melhoria contínua, para criação e administração de novos recursos na organização, as habilidades de cognição, inteligência, autonomia, aprendizagem e gestão do conhecimento organizacional representam fontes do desenvolvimento de competências essenciais dinâmicas na organização. Por conseguinte, com base nos conceitos de recursos e competências dinâmicas apresentadas em Hitt et al. (1999) e Lei et al. (1996), este artigo sugere que as habilidades organizacionais propostas podem beneficiar a organização com vantagem competitiva.

\subsubsection{Competências essenciais}

Competências essenciais podem ser compreendidas como conjuntos de conhecimento tácito e coletivo, que são desenvolvidos por meio de processos de aprendizagem, e que constituem fontes de inovação, criação de valor e de benefícios para os clientes e do desenvolvimento da vantagem competitiva da organização (Lei et al., 1996; Oliveira, 1999). Adicionalmente, competências essenciais se caracterizam como não fáceis de serem imitadas ou reproduzidas por competidores. Este artigo concorda com estas definições e apresenta uma reestruturação e extensão das mesmas considerando três aspectos fundamentais: primeiro, ao assumir que competências essenciais podem ser conjuntos de conhecimento tácito e explícito, e que há conversão entre conhecimento tácito e explícito de acordo com o modelo SECl (Socialização, Externalização, Combinação e Internalização) apresentado em Takeuchi \& Nonaka (2004); segundo, ao considerar que o conhecimento é criado inicialmente na mente dos indivíduos e em seguida expandido e cristalizado nos níveis coletivos de grupos e organizacionais, seguindo, portanto, os processos do ciclo espiral de criação do conhecimento apresentado em Takeuchi \& Nonaka (2004); e terceiro, ao compreender que as competências essenciais, e, portanto, os conjuntos de conhecimento na organização, são desenvolvidos principalmente através de processos cognitivos ou habilidades cognitivas na organização, incluindo as habilidades de cognição, inteligência, autonomia, aprendizagem e gestão do conhecimento organizacional. A inclusão do conhecimento explícito na definição de competências essenciais se faz necessária uma vez que a expansão do conhecimento individual para o coletivo na organização depende essencialmente da conversão entre o conhecimento tácito e explícito, atendendo aos quatro modos de conversão do conhecimento denominados socialização (de tácito para tácito), externalização (de tácito para explícito), combinação (de explícito para explícito) e internalização (de explícito para implícito) (Takeuchi $\&$ Nonaka, 2004).

\subsection{Modelo estratégico dinâmico}

A Figura 1 ilustra a proposta de um modelo de processo dinâmico de criação de vantagem competitiva sustentadora da organização. Neste modelo, as linhas que ligam os elementos entre si indicam que a evolução e melhoria de cada elemento influenciam e contribuem para o desenvolvimento de cada um dos outros elementos. 0 seu funcionamento apresenta as seguintes características:

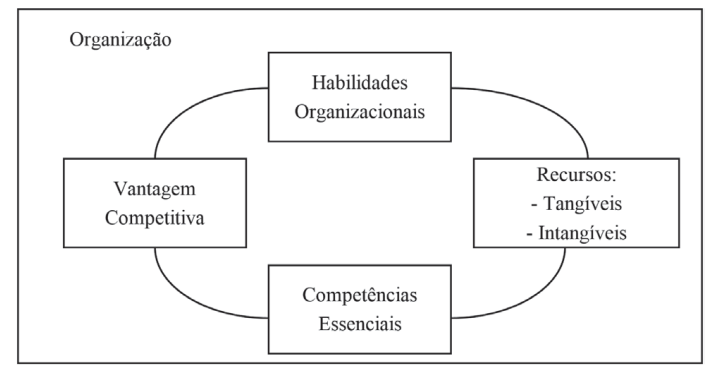

Figura 1. Modelo dinâmico de criação de vantagem competitiva. Fonte: Elaborado pelos autores (2015). 
(1) Primeiramente, utilizam-se as habilidades organizacionais existentes para a administração estratégica dos recursos na organização, que, por conseguinte, contribuem para a criação de novas habilidades organizacionais.

(2) Em seguida, as habilidades organizacionais, que envolvem habilidades de cognição, inteligência, autonomia, aprendizagem e gestão do conhecimento, contribuem para o desenvolvimento das competências essenciais, e estas competências formam a base de criação e sustentação da vantagem competitiva da organização.

(3) Adicionalmente, este modelo dinâmico considera que estímulos e variações no ambiente poderão afetar a vantagem competitiva da organização, e, por conseguinte, irão ativar ou impulsionar as habilidades organizacionais, iniciando novos ciclos do desenvolvimento de competências essenciais e de criação da vantagem competitiva sustentadora da organização.

A seguir são propostos conceitos e princípios de cognição organizacional e realizadas revisões sobre os conceitos de inteligência, autonomia, aprendizagem e gestão do conhecimento. Deve-se destacar que o intuito das revisões é contribuir para definição das fronteiras entre cognição organizacional e as demais habilidades na organização.

\subsection{Cognição organizacional}

\subsubsection{Cognição humana e organizacional}

Organismos do sistema ecológico ou natural têm apresentado evoluções e melhorias nas suas habilidades e mecanismos de adaptação no ambiente. Entre tais organismos, o ser humano se destaca como a espécie que desenvolveu e atingiu a maior probabilidade de sobrevivência e reprodução, dando continuidade à sua existência e evolução. Esta predominância dos seres humanos é essencialmente privilegiada pela evolução de seu cérebro, emoções e processos cognitivos (Heyes \& Huber, 2000; Simon, 1983). Entre os resultados desta trajetória evolucionária do ser humano incluem suas habilidades de busca de informação, organização do conhecimento, tomada de decisão, aprendizagem e resolução de problemas. Seres humanos se adaptam ao ambiente, mas também mudam o ambiente em favor de suas necessidades e em busca do benefício próprio. Ao realizarem atividades como o cultivo da terra pela agricultura, o aperfeiçoamento do sistema imunológico pela medicina, a exploração de recursos naturais para produção de energia, a construção de cidades, a elaboração de legislações, o desenvolvimento de sistemas de transporte e de telecomunicações, o desenho de novos empreendimentos, o projeto de máquinas que mimetizam seu próprio comportamento, entre outras tarefas, pode-se entender que, neste contínuo de evolução, os seres humanos têm transferido suas habilidades para os sistemas, e, em especial, para as organizações (Nobre et al., 2009a). Certamente, entre as principais motivações para se organizar, pode-se mencionar que as organizações beneficiam os seres humanos ao expandirem suas limitações cognitivas, físicas, temporais, institucionais e espaciais (Carley \& Gasser, 1999; Nobre et al., 2009a).

Sob este panorama, observa-se que, enquanto a cognição humana pode ser entendida como parte do sistema natural e evolutivo, a cognição organizacional pode ser mais bem compreendida como parte de um sistema artificial que envolve o conceito de projeto (Simon, 1996). Por conseguinte, a habilidade cognitiva na organização pode ser alterada e aperfeiçoada através de processos de mudança e projeto organizacionais. Consequentemente, cognição organizacional, ou, mais precisamente, o grau de cognição na organização é função da escolha de seus elementos (estrutura social, objetivos, tecnologia e participantes) e também dependente de seu ambiente.

\subsubsection{Dez princípios da cognição organizacional}

Princípios sobre cognição organizacional foram propostos em Nobre et al. (2009a) sob uma perspectiva de processamento de informação. Esta seção contribui através da reestruturação e extensão desses princípios para um contexto mais avançado que abrange perspectivas computacionais e interpretativas, onde o conhecimento, as habilidades organizacionais e o contexto estratégico recebem maior ênfase.

P1) Cognição envolve processos e representações que contribuem para proporcionarem as habilidades de inteligência, autonomia, aprendizagem e gestão do conhecimento aos indivíduos, grupos e organizações. Portanto, a cognição pode ser compreendida como uma habilidade estratégica.

P2) A cognição organizacional está associada a processos e representações na organização. No nível individual, os processos cognitivos e as representações mentais contribuem para a criação do conhecimento tácito e explícito dos participantes na organização; e, nos níveis de grupo e organizacional, os processos e as representações contribuem para a criação do conhecimento tácito e explícito coletivos, que são expandidos e cristalizados em toda a organização. A expansão do conhecimento na organização pode seguir o modelo ou ciclo espiral de criação do conhecimento apresentado em lchijo \& Nonaka (2006). 
P3) Os principais agentes de cognição organizacional são os participantes na organização e suas redes sociais ou coletivas. Sob essa perspectiva, a cognição emerge de mentes individuais e coletivas, e, portanto, de fenômenos e processos individuais e sociais. Agentes de cognição organizacional incluem pessoas e máquinas cognitivas (Nobre et al., 2009a, c).

P4) A estrutura social, os objetivos, a tecnologia e a estratégia da organização são elementos-chave que influenciam e auxiliam os participantes na criação de processos cognitivos e representações na organização.

P5) Cognição organizacional é também influenciada por processos interorganizacionais e pelo ambiente.

P6) Cognição organizacional pode ser associada a graus de cognição na organização, onde o termo grau de cognição pode ser simbolicamente associado a medidas tangíveis e intangíveis de processos cognitivos e representações na organização.

P7) Estudos sobre cognição organizacional são importantes e necessários quando se requer o projeto de organizações que apresentem alto grau de cognição para administração de incertezas ambientais.

P8) 0 grau de cognição organizacional depende da escolha de modelos de organização, e, por conseguinte, da seleção dos elementos da organização, incluindo estrutura social, objetivos, tecnologias, participantes, além da estratégia.

P9) A escolha dos elementos da organização depende do ambiente. Consequentemente, cognição organizacional (e o grau de cognição organizacional) se torna contingente ao ambiente (e ao nível de incerteza ambiental).

P10) Cognição é uma habilidade estratégica na organização que contribui para o desenvolvimento das competências essenciais, e, por conseguinte, que proporciona a base para criação de vantagem competitiva sustentadora da organização.

\subsection{Inteligência organizacional}

Inteligência pode ser compreendida como uma habilidade mental generalizada (Schmidt \& Hunter, 2000), a qual depende de processos racionais e emocionais (Goleman, 1994). Racionalidade ou processos racionais representam a habilidade de seguir procedimentos para tomada de decisão e resolução de problema, com o intuito de satisfazer critérios (satisfice) e atingir objetivos (Simon, 1997a). Quando indivíduos obtêm resultados satisfatórios através de processos racionais, pode-se associar racionalidade à inteligência. Processos emocionais (Scherer, 1982) são menos procedimentais que racionalidade e não são propositalmente utilizados para realizar objetivos. Contudo, alguns pesquisadores têm demonstrado que emoções exercem um papel importante ao motivar pessoas e direcionar suas ações na busca de objetivos (Bagozzi et al., 1998; Keltner \& Gross, 1999; Keltner \& Haidt, 1999). Quando processos emocionais proporcionam aos indivíduos a capacidade de se tornarem bem-sucedidos, a emoção pode ser associada à inteligência. De uma forma complementar, enquanto a emoção influencia processos cognitivos tais como atenção, aprendizagem, tomada de decisão e resolução de problemas (Goleman, 1994), a cognição atua a favor da emoção em processos de interpretação de estímulos (Plutchik, 1982), criação de significado e controle de processos e estados emocionais (Nobre et al., 2009a). Portanto, inteligência, e, em particular, comportamento inteligente, depende de processos cognitivos e emocionais.

Inteligência organizacional pode também ser associada a graus de inteligência na organização. Apesar de apresentar algumas similaridades e fronteiras entre si, os campos da cognição e inteligência organizacionais se distinguem pelo fato de que enquanto a cognição organizacional está associada a processos cognitivos e representações na organização, a inteligência organizacional está relacionada ao grau ou intensidade com que a organização satisfaz, ou seja, satisfice (Simon, 1997b), seus objetivos. Portanto, pode-se dizer que quanto maior o grau de cognição na organização, maior é a sua chance de exibir comportamento inteligente (Nobre et al., 2009a, b).

\subsection{Autonomia organizacional}

Autonomia representa a habilidade de indivíduos, grupos e organizações de atuar e agir através da cognição. Organismos autônomos estão continuamente buscando formas de ampliar sua independência intelectual, e, portanto, estes organismos se concentram em atividades que possam melhorar as suas habilidades cognitivas. As autonomias de indivíduos, grupos e organizações também dependem das interações destes organismos com o ambiente para troca e renovação de recursos, sendo que suas autonomias podem ser ampliadas à medida que estes organismos adquirem, processam, criam, armazenam, distribuem e aplicam novos recursos em benefício próprio. Autonomia organizacional também pode ser associada a grau de autonomia na organização. Sob esta perspectiva, pode-se dizer que quanto maior o grau de cognição na organização, maior é o grau de autonomia organizacional (Nobre et al., 2009a, b). 


\subsection{Aprendizagem organizacional}

Aprendizagem organizacional pode ser associada à criação, armazenagem e transferência do conhecimento nas organizações (Argote, 2007; Dierkes et al., 2003). $\mathrm{Na}$ psicologia, aprendizagem pode ser entendida como processo de realizar mudanças na mente e no comportamento de indivíduos, incluindo alterações de perspectivas, valores, habilidades e conhecimento, através de experiências, influências Ambientais, atividades cognitivas e emocionais (Bernstein et al., 2008; llleris, 2007; Lefrançoies, 1995; Minsky, 1986; Reed, 1988). Este artigo apoia essa perspectiva da psicologia e define aprendizagem organizacional como o processo de realizar mudanças nos elementos e no comportamento da organização através de experiências, cognição, emoção (Fineman, 2000) e influências ambientais, em benefício da organização. Essa definição sugere a existência de relações entre aprendizagem e cognição organizacional. Por um lado, é plausível dizer que a aprendizagem organizacional afeta a cognição organizacional, e, mais especificamente, o grau de cognição organizacional, pela mudança nos processos cognitivos e representações na organização. Por outro lado, é também plausível considerar que a aprendizagem organizacional depende da cognição organizacional, e, mais precisamente, de processos cognitivos e representações, para a efetiva realização de mudanças, criação e administração do conhecimento organizacional. Processos de mudança na organização estão fundamentados em mecanismos e modelos que, em grande parte, são adaptações e extensões de conceitos e princípios de retroalimentação, controle adaptativo e sistemas de aprendizagem os quais tiveram suas origens nos campos da cibernética e teoria geral dos sistemas (Ashby, 1968; Von Bertalanffy, 1968; Buckley, 1968; Wiener, 1961). Os modelos de aprendizagem organizacional mais bem conhecidos incluem circuitos de tipos simples e duplo (Argyris \& Schön, 1978), além de meta-aprendizagem, cujo conceito foi apresentado em Biggs (1985) para descrever o estado de consciência e autocontrole do aprendiz sobre seu próprio processo de aprendizagem. Estudos adicionais sobre meta-aprendizagem e sua distinção de dêutero-aprendizagem e aprendizagem planejada são apresentados em Visser (2007), e o emprego da meta-aprendizagem no desenvolvimento de competências essenciais dinâmicas na organização é proposto em Lei et al. (1996).

Sob esta perspectiva, cognição é uma habilidade que proporciona aos indivíduos, grupos e organizações a capacidade de aprendizagem.

\subsection{Gestão do conhecimento}

0 conceito de gestão do conhecimento depende da perspectiva adotada sobre o conhecimento. Neste artigo, o conhecimento é originado a partir do processo de personalização da informação na mente de indivíduos, envolvendo a aquisição e interpretação da informação e a criação de significado (Alavi \& Leidner, 2001). Adicionalmente, o conhecimento pode ser classificado de acordo com dimensões epistemológicas e ontológicas (1chijo \& Nonaka, 2006). A partir destes preceitos, este artigo define que gestão do conhecimento organizacional abrange um conjunto de práticas coletivas e processos sociais para (1) criação, incluindo renovação e conversão (do conhecimento tácito para explícito, e de explícito para tácito), (2) armazenagem e busca, (3) transferência e distribuição, e (4) aplicação do conhecimento, em benefício da organização. Enquanto os indivíduos ou participantes são os agentes primários de criação do conhecimento, a organização é a agência que os auxilia e proporciona as condições e recursos necessários para o desenvolvimento, aplicação e expansão do conhecimento para as camadas ontológicas mais elevadas, nos níveis de grupo, organizacional e interorganizacional (Takeuchi \& Nonaka, 2004). Os campos da gestão do conhecimento e da cognição organizacional compartilham fronteiras sobre a produção e administração do conhecimento. Neste contexto, cognição organizacional contribui para a gestão do conhecimento nas organizações através de processos que incluem formação de conceitos, categorização, representação e organização do conhecimento, memória, aprendizagem, entre outros.

Mais recentemente, a gestão do conhecimento passou a ser incorporada e tratada como um ramo da administração estratégica baseada em recursos, sendo que, neste contexto, o conhecimento é compreendido como o principal ativo estratégico na formação de uma teoria da firma baseada no conhecimento (Grant, 1996; Ichijo \& Nonaka, 2006; Oliveira, 1999; Spender, 1996a, b). A partir desta nova perspectiva, estudos sobre a gestão do conhecimento organizacional têm expandido suas contribuições e ganhado uma conotação mais pragmática dentro da área de teoria das organizações, e têm sido também relacionados ao conceito de inovação organizacional e tecnológica (Fleury \& Fleury, 1997; Fleury \& Oliveira Junior, 2001; Takahashi \& Takahashi, 2007). Com base nesses avanços em gestão do conhecimento, este artigo sugere que cognição organizacional pode ser compreendida como uma habilidade estratégica na organização, e, portanto, propõe a sua inserção num contexto de uma teoria da empresa baseada em habilidades cognitivas. 


\section{Relações entre cognição organizacional e incerteza ambiental}

Esta seção contribui com conceitos incipientes para a formação de uma relação contingencial entre cognição organizacional e incerteza ambiental que poderá ser colocada à prova em pesquisas futuras através de estudos aplicados. As definições e proposições introduzidas nesta seção estão fundamentadas em teorias da contingência (Burns \& Stalker, 1994; Donaldson, 2001; Galbraith, 1977; Lawrence, 2000), administração estratégica e visão da empresa baseada em recursos (Hitt et al., 1999; Prahalad \& Hamel, 1990; Wernerfelt, 1984). Contudo, este artigo estende esses trabalhos ao introduzir a cognição como a principal habilidade estratégica que contribui para o controle, governo e redução do nível de incerteza ambiental. Consequentemente, com a redução do nível de incerteza, este artigo reforça a proposição de que a cognição organizacional contribui para a criação da vantagem competitiva da organização.

Anteriormente à descrição das relações contingenciais, esta seção apresenta definições de Nível Relativo de Incerteza Ambiental e Grau Relativo de Cognição Organizacional.

\subsection{Nível relativo de incerteza ambiental}

Definição 1: 0 Nível Relativo de Incerteza Ambiental $\left(R_{\nu}\right)$ é simbolicamente definido pela proporção entre o Nível de Incerteza Ambiental $\left(E_{l}\right)$ e o Grau de Cognição Organizacional $(O)$, sendo que, $R_{U}=E_{U} / O_{C}$

A definição (1) indica que o Nível Relativo de Incerteza Ambiental $\left(R_{v}\right)$ varia de acordo com as seguintes causalidades:

(i) $R_{U}$ reduz quando $O_{C}$ aumenta, para um dado $E_{U}$.

(ii) $R_{U}$ reduz quando $E_{U}$ reduz, para um dado $O_{C}$

(iii) $R_{U}$ aumenta quando $O_{C}$ reduz, para um dado $E_{U}$

(iv) $R_{U}$ aumenta quando $E_{U}$ aumenta, para um dado $O_{c}$

\subsection{Grau relativo de cognição organizacional}

Definição 2: 0 Grau Relativo de Cognição Organizacional $\left(R_{c}\right)$ é simbolicamente definido pela proporção entre o Grau de Cognição Organizacional $(O)$ e o Nível de Incerteza Ambiental $\left(E_{\nu}\right)$, sendo que, $R_{c}=O_{c} / E_{u}$

Similarmente, a definição (2) indica que o Grau Relativo de Cognição Organizacional $\left(R_{c}\right)$ varia de acordo com as seguintes causalidades: (a) $R_{C}$ aumenta quando $O_{C}$ aumenta, para um dado $E_{U}$.

(b) $R_{C}$ aumenta quando $E_{U}$ reduz, para um dado $O_{C}$.

(c) $R_{C}$ reduz quando $O_{C}$ reduz, para um dado $E_{U}$.

(d) $R_{C}$ reduz quando $E_{U}$ aumenta, para um dado $O_{C}$.

\subsection{Análise das relações}

As definições (1) e (2) e suas respectivas causalidades (i) a (iv), e (a) a (d) sugerem um total de oito alternativas de estratégias que podem levar a quatro objetivos ou resultados possíveis: a redução ou aumento no Nível Relativo de Incerteza Ambiental $\left(R_{U}\right)$, e a redução ou aumento no Grau Relativo de Cognição Organizacional $(R)$. Como o foco deste artigo é sobre a cognição organizacional, selecionaram-se as estratégias (i) e (a), pois ambas alternativas aumentam o Grau de Cognição Organizacional $\left(O_{C}\right)$ ocasionando redução no Nível Relativo de Incerteza Ambiental $\left(R_{l}\right)$ e aumento no Grau Relativo de Cognição Organizacional $\left(R_{c}\right)$. Com base nestas definições, propõe-se que:

Proposição 1: Quanto maior o Grau de Cognição Organizacional $(O)$, menor o Nível Relativo de Incerteza Ambiental $\left(R_{\nu}\right)$, e maior o Grau Relativo de Cognição Organizacional $\left(R_{C}\right)$.

A proposição (1) sugere que, enquanto o ambiente influencia a organização através de informação e incerteza, a organização pode regular ou controlar o ambiente através de suas habilidades que incluem cognição, inteligência, autonomia, aprendizagem e gestão do conhecimento (Nobre \& Walker, 2011). Outra importante conclusão derivada da proposição (1) se refere à relação entre a cognição organizacional e a vantagem competitiva da organização.

\subsection{Relação entre cognição organizacional e vantagem competitiva}

Resultados de análises na literatura mostram que as organizações com habilidades de navegação em ambientes desconhecidos e de administração de um alto nível de incerteza são aquelas com melhores condições de desenvolverem novas competências e de criação de vantagem competitiva (Hitt et al., 2008; Venkatraman \& Subramaniam, 2002). Este artigo apoia esses resultados e propõe que as habilidades de cognição, inteligência, autonomia, aprendizagem e gestão do conhecimento contribuem para a administração de incertezas ambientais, o desenvolvimento de competências essenciais e a criação de vantagem competitiva sustentadora. A partir desses resultados 
de literatura e dos conceitos propostos neste trabalho, propõe-se que:

Proposição 2: Quanto maior o Grau de Cognição Organizacional, menor o Nível Relativo de Incerteza Ambiental, e maior a Vantagem Competitiva da Organização.

\subsection{Implicações teóricas e práticas}

Os princípios $\mathrm{P} 4, \mathrm{P} 5, \mathrm{P} 8$ e P9 da cognição organizacional e as proposições 1 e 2 introduziram uma noção de contingência entre a cognição e o ambiente, e apontaram para uma visão contingencial entre cognição organizacional, incerteza ambiental e vantagem competitiva. A Figura 2 ilustra uma visão contingencial em duas dimensões que se subdividem em quatro quadrantes ou quatro modos de operação da organização.

0 primeiro quadrante caracteriza o tipo de organização que é foco deste artigo, ou seja, que concentra suas estratégias na busca de altos graus de cognição organizacional para a administração de altos níveis de incerteza ambiental. Esta classe atende aos critérios de Sistemas Centrados no Cliente (Nobre, 2011) e de organizações inovadoras (Nobre et al., 2012). De acordo com este quadrante, pode-se dizer que indivíduos, grupos e organizações que possuem um alto grau de cognição tenderão a apresentar melhores desempenhos em ambientes de alto nível de incerteza ou complexidade. Adicionalmente, com base na Lei de Variedade de Requisito de Ashby (1958), Calori et al. (1994) propõem que executivos que atuam em ambientes ou mercados complexos, caracterizados por exemplo por uma alta variedade de negócios e diversidade geográfica, tendem a desenvolver mais habilidades e interpretações diferenciadas de eventos e situações, resultando em mapas cognitivos mais elaborados e complexos, consistentes com a complexidade situacional ou ambiental. Portanto, processos cognitivos e representações mais elaboradas deverão contribuir para atenuar a incerteza caracterizada pela escassez de informações e por condições técnicas, sociais, geográficas, políticas e econômicas do ambiente.

0 segundo quadrante caracteriza organizações denominadas obsoletas por estarem operando em situação de incompatibilidade entre um baixo grau de cognição e um alto nível de incerteza ambiental. Estas organizações tenderão a apresentar um baixo nível de desempenho ou um curto ciclo de vida. Organizações com baixo nível de cognição apresentarão deficiências de inteligência, autonomia, aprendizagem e gestão do conhecimento, não sendo, portanto, promotoras da inovação.

0 terceiro quadrante ilustra organizações com baixo grau de cognição e que operam em ambientes técnicos simples e mercados com baixa demanda por inovação. Estas organizações tendem a sobreviver, por exemplo, em mercados protegidos por sistemas regulatórios e fechados, ou ainda em mercados de regiões e países inicialmente não servidos; onde mercados não servidos incluem aqueles na base da pirâmide (Prahalad, 2004).

0 quarto quadrante envolve organizações com alto grau de cognição em ambientes simples ou com baixo nível de incerteza. Embora estas organizações possuam um nível de capacidades mais elevado que aquelas demandadas pelo seu ambiente atual, elas apresentam habilidades e estratégias que as capacitam a se adaptarem a ambientes mais complexos.

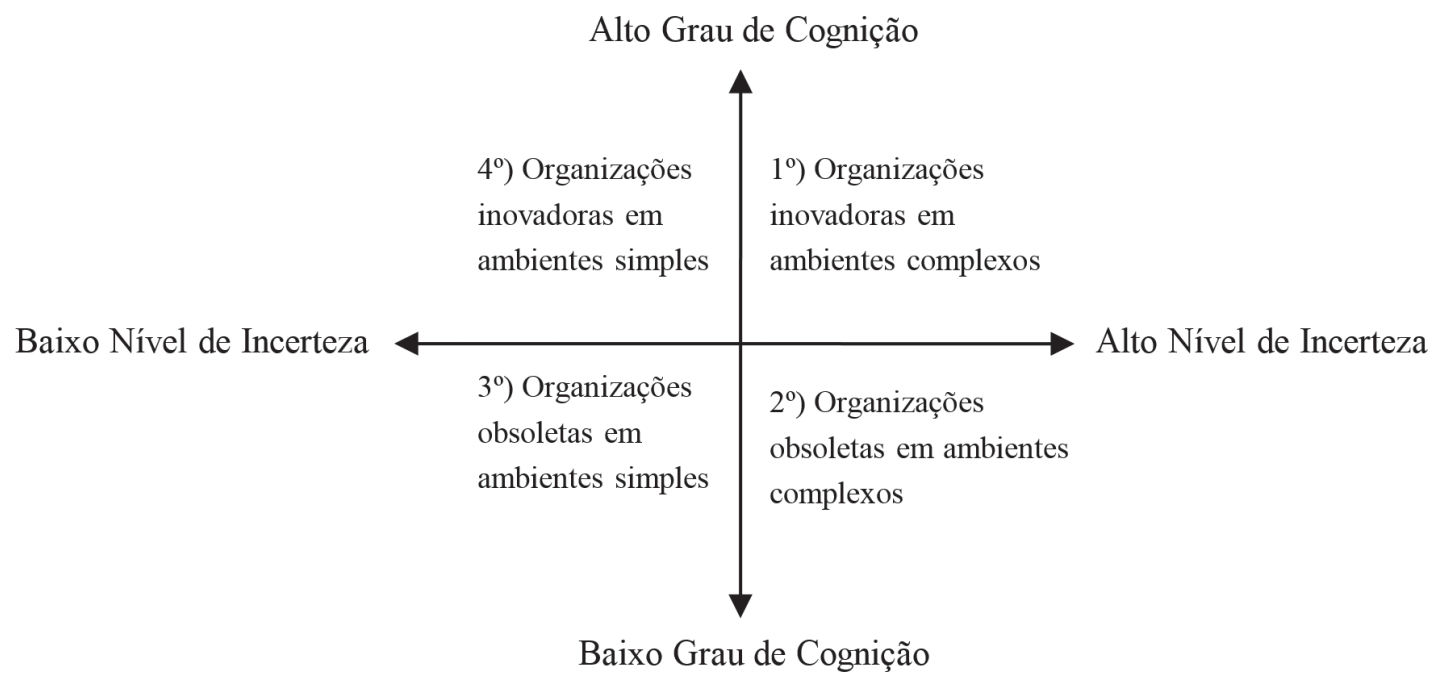

Figura 2. Visão contingencial: cognição vs. Incerteza. Fonte: Elaborado pelos autores (2015). 


\section{Conclusões}

Este trabalho contribuiu para o campo da cognição organizacional através de revisão de literatura, conceitualizações e a sua introdução no contexto da administração de recursos estratégicos.

Em sua proposta, este trabalho contribuiu com quatro importantes perspectivas, distintas e complementares, sobre cognição organizacional. Primeiro, ao compreender cognição organizacional como um conjunto de processos e representações na organização, sendo esta perspectiva uma integração das abordagens computacionais e interpretativas. Segundo, ao definir cognição como a habilidade principal que proporciona inteligência, autonomia, aprendizagem e gestão do conhecimento aos indivíduos, grupos e organizações. Esta perspectiva contribui ao sugerir a formação de uma base de preceitos sobre uma teoria de habilidades organizacionais, além de esclarecer relações complementares entre cognição e os conceitos de inteligência, autonomia, aprendizagem e gestão do conhecimento organizacional. Terceiro, ao apresentar cognição organizacional como uma habilidade estratégica na organização que contribui para o desenvolvimento das competências essenciais, e, por conseguinte, que proporciona a criação de vantagem competitiva sustentadora da organização. Esta perspectiva está fundamentada na proposta do modelo estratégico dinâmico que explicou a interação entre os conceitos de recursos estratégicos, habilidades organizacionais e competências essenciais para a criação de vantagem competitiva sustentadora da organização. Portanto, nesta perspectiva, a cognição organizacional foi introduzida em um contexto estratégico, e, por conseguinte, mais pragmático, contribuindo para a redução entre teoria e prática. Ainda sob este panorama, este trabalho sugeriu que cognição organizacional pode ser ainda estudada num contexto de uma teoria da empresa baseada em habilidades cognitivas e organizacionais. Quarto, ao estabelecer relações contingenciais de causalidade entre cognição organizacional e incerteza ambiental. Esta perspectiva contribuiu ao definir cognição como a principal habilidade estratégica na organização capaz de controlar, governar e reduzir o nível de incerteza no ambiente. Desta forma, foram definidas perspectivas de organizações que priorizam a busca de altos graus de cognição organizacional para a administração de altos níveis de incerteza ambiental e criação de vantagem competitiva sustentadora.

Este artigo se resumiu em um estudo teórico e como proposta de sua extensão e melhoria, sugere-se o teste empírico das proposições apresentadas através de estudo de casos múltiplos ou de levantamentos (surveys) com empresas de diversos setores industriais de forma a sustentar uma teoria contingencial como ilustrada na Figura 2 sobre cognição e incerteza. Adicionalmente, um estudo empírico com uma população bem representativa poderia sugerir se determinados setores industriais tendem ou não a possuir empresas com graus mais elevados de cognição que empresas de outros setores.

\section{Referências}

Alavi, M., \& Leidner, D. E. (2001). Knowledge management and knowledge management systems: conceptual foundations and research issues. Management Information Systems Quarterly, 25(1), 107-136. http://dx.doi.org/10.2307/3250961.

Argote, L. (2007). Organizational learning: creating, retaining and transferring knowledge. New York: Springer.

Argyris, C. (1999). On organizational learning. Oxford: Blackwell Publishing.

Argyris, C., \& Schön, D. A. (1978). Organizational learning: a theory of action perspective. Reading: Addison-Wesley.

Ashby, W. R. (1958). Requisite variety and its implications for the control of complex systems. Cybernetica, 1(2), 83-99.

Ashby, W. R. (1968). Principles of the self-organizing system. In W. Buckley (Ed.), Modern systems research for the behavioral scientist (pp. 108-118). Chicago: Aldine Publishing Company.

Bagozzi, R. P., Baumgartner, H., \& Pieters, R. (1998). Goaldirected emotions. Cognition and Emotion, 12(1), 1-26. http://dx.doi.org/10.1080/026999398379754.

Bailey, K. D. (1982). Methods of social research. New York: The Free Press.

Berger, P. L., \& Luckmann, T. (1967). The social construction of reality: a treatise in the sociology of knowledge. Harmondsworth: Penguin Books.

Bernstein, D. A., Penner, L. A., Clarke-Stewart, A., \& Roy, E. J. (2008). Psychology (8. ed.). Boston: Houghton Mifflin Company.

Biggs, J. B. (1985). The role of meta-learning in study process. The British Journal of Educational Psychology, 55(3), 185212. http://dx.doi.org/10.1111/j.2044-8279.1985.tb02625.x.

Black, M. (1937). Vagueness: an exercise to logical analysis. Philosophy of Science, 4(4), 427-455. http://dx.doi. org/10.1086/286476.

Black, M. (1963). Reasoning with loose concepts. Dialogue, 2(1), 1-12. http://dx.doi.org/10.1017/S001221730004083X.

Bless, H., Fiedler, K., \& Strack, F. (2004). Social cognition: how individuals construct reality (Social Psychology: a Modular Course). New York: Psychology Press.

Brewer, M. B., \& Hewstone, M. (2004). Social cognition. Oxford: Blackwell Publishing.

Buckley, W. (1968). Modern systems research for the behavioral scientist. Chicago: Aldine Publishing Company.

Burns, T., \& Stalker, G. M. (1994). The management of innovation. Oxford: Oxford University Press.

Calori, R., Johnson, G., \& Sarnin, P. (1994). CEO's cognitive maps and the scope of the organization. Strategic Management Journal, 15(6), 437-457. http://dx.doi.org/10.1002/ smj.4250150603.

Carley, K. M., \& Gasser, L. (1999). Computational organizational theory. In G. Weiss (Ed.), Multiagent systems: a modern 
approach to distributed artificial intelligence (pp. 299-330). Cambridge: MIT Press.

Choo, C. W. (2005). The knowing organization: how organizations use information to construct meaning, create knowledge, and make decisions (2. ed.). Oxford: Oxford University Press.

Cyert, R. M., \& March, J. G. (1963). A behavioral theory of the firm. Oxford: Blackwell Publishers.

Dierkes, M., Antal, A. B., Child, J., \& Nonaka, I. (2003). Handbook of organizational learning and knowledge. Oxford: Oxford University Press.

Donaldson, L. (2001). The contingency theory of organizations (Foundations for Organizational Science). London: Sage.

Ducan, R. B. (1972). Characteristics of organizational environments and perceived environmental uncertainty. Administrative Science Quarterly, 17(3), 313-327. http:// dx.doi.org/10.2307/2392145.

Eden, C., \& Spender, J. C. (1998). Managerial and organizational cognition: theory, methods and research. London: Sage.

Eysenck, M. W., \& Keane, M. T. (2005). Cognitive psychology: a student's handbook. London: Psychology Press.

Fineman, S. (2000). Emotion in organizations (2. ed.). London: Sage.

Fleury, A., \& Fleury, M. T. L. (1997). Aprendizagem e inovação organizacional: as experiências de Japão, Coreia e Brasil (2. ed.). São Paulo: Atlas.

Fleury, M. T. L., \& Oliveira Junior, M. M., (2001). Gestão estratégica do conhecimento: integrando aprendizagem, conhecimento e competências. São Paulo: Atlas.

Galbraith, J. R. (1977). Organization design. Reading: AddisonWesley.

Galbraith, J. R. (2002). Designing organizations: an executive guide to strategy, structure, and process. San Francisco: Jossey-Bass.

Goleman, D. (1994). Emotional intelligence: why it can matter more than IQ. New York: Bantam Books.

Grant, R. M. (1996). Toward a knowledge-based theory of the firm. Strategic Management Journal, 17(S2), 109-122. http://dx.doi.org/10.1002/smj.4250171110.

Gurvitch, G. (1971). The social frameworks of knowledge. Oxford: Blackwell.

Harvard Business Review. (1998). Harvard business review on knowledge management. Boston: Harvard Business School Press.

Heyes, C., \& Huber, L. (2000). The evolution of cognition. Cambridge: MIT Press.

Hitt, A., Clifford, P. G., Nixon, R. D., \& Coyne, K. P. (1999). Dynamic strategic resources: development, diffusion and integration. Chichester: Wiley.

Hitt, M. A., Ireland, R. D., \& Hoskisson, R. E. (2008). Strategic management: competitiveness and globalization, concepts and cases. Cincinnati: South-Western College Pub.

Hodgkinson, G. P., \& Healey, M. P. (2008). Cognition in organizations. Annual Review of Psychology, 59(1), 387-417. http://dx.doi.org/10.1146/annurev.psych.59.103006.093612. PMid:17547531.

Holzner, B., \& Marx, J. (1979). The knowledge application: the knowledge system in society. Boston: Allyn \& Bacon. landoli, L., \& Zollo, G. (2007). Organizational cognition and learning: building systems for the learning organization. New York: Information Science.

lchijo, K., \& Nonaka, 1. (2006). Knowledge creation and management: new challenges for managers. Oxford: Oxford University Press.

Illeris, K. (2007). How we learn: learning and non-learning in school and beyond. London: Routledge.

Katz, D., \& Kahn, R. L. (1967). The social psychology of organizations. New York: John Wiley \& Sons.

Keltner, D., \& Gross, J. (1999). Functional accounts of emotions. Cognition and Emotion, 13(5), 467-480. http://dx.doi. org/10.1080/026999399379140.

Keltner, D., \& Haidt, H. (1999). Social functions of emotions at four levels of analysis. Cognition and Emotion, 13(5), 505-521. http://dx.doi.org/10.1080/026999399379168.

Klir, G. J., \& Folger, T. A. (1988). Fuzzy sets, uncertainty, and information. Englewood Cliffs: Prentice Hall.

Lant, T. K., \& Shapira, Z. (2001). Organizational cognition: computation and interpretation. London: Lawrence Erlbaum Associates.

Lawrence, P. R. (2000). The contingency approach to organizational design. In R. T. Golembiewski (Ed.), Handbook of organizational behavior (2. ed.). Boca Raton: CRC Press.

Lefrançoies, G. (1995). Theories of human learning. Pacific Grove: Brooks Cole Publishing Company.

Lei, D., Hitt, M. A., \& Bettis, R. (1996). Dynamic core competences through meta-learning and strategic context. Journal of Management, 22(4), 549-569. http://dx.doi. org/10.1177/014920639602200402.

March, J. G., \& Simon, H. A. (1958). Organizations. New York: John Wiley \& Sons.

Minsky, M. (1986). The society of mind. London: Picador.

Newell, A. (1990). Unified theories of cognition. Cambridge: Harvard University.

Newell, A., \& Simon, H. A. (1972). Human problem solving. Englewood Cliffs: Prentice-Hall.

Nicolini, D. (1999). Comparing methods for mapping organizational cognition. Organization Studies, 20(5), 833-860. http:// dx.doi.org/10.1177/0170840699205006.

Nobre, F. S. (2011). Core competencies of the new industrial organization. Journal of Manufacturing Technology Management, 22(4), 422-443. http://dx.doi. org/10.1108/17410381111126391.

Nobre, F. S., \& Ribeiro, R. E. M. (2013). Sustentabilidade e cognição: estudo de casos múltiplos no índice de sustentabilidade empresarial da BM\&FBovespa. Revista de Administração Contemporânea, 17(4), 499-517. http:// dx.doi.org/10.1590/S1415-65552013000400007.

Nobre, F. S., \& Walker, D. (2011). An ability-based view of the organization: strategic-resource and contingency domains. The Learning Organization, 18(4), 333-345. http://dx.doi. org/10.1108/09696471111132531.

Nobre, F. S., Tobias, A. M., \& Walker, D. (2009a). Organizational and technological implications of cognitive machines: designing future information management systems. New York: IGl Global.

Nobre, F. S., Tobias, A. M., \& Walker, D. (2009b). The pursuit of cognition in manufacturing organizations. Journal of Manufacturing Systems, 27(3), 145-157. 
Nobre, F. S., Tobias, A. M., \& Walker, D. (2009c). The Impact of cognitive machines in complex decisions and organizational change. $A l$ \& Society, 24(4), 365-381. http://dx.doi. org/10.1007/s00146-009-0207-4.

Nobre, F. S., Walker, D., \& Brown, M. (2014). Ability-based view in action: a software corporation study. Brazilian Administration Review, 11(2), 164-187.

Nobre, F. S., Walker, D., \& Harris, R. (2012). Technological, managerial and organizational core competencies: dynamic innovation and sustainable development. New York: IGl Global.

Nonaka, 1. (2005). Knowledge management: critical perspectives on business and management (Vol. 1, 2 \& 3). London: Routledge Falmer.

Oliveira, J. (1999). Core competenies and the knowledge of the firm. In A. Hitt, P. G. Clifford, R. D. Nixon \& K. P. Coyne (Eds.), Dynamic strategic resources: development, diffusion and integration (Chap. 2, pp. 17-42). New Jersey: Wiley.

Pine, B. J. (1999). Mass customization: the new frontier in business competition. Boston: Harvard Business School.

Plutchik, R. (1982). A psychoevolutionary theory of emotions. Social Sciences Information. Information Sur les Sciences Sociales, 21(4-5), 529-553. http://dx.doi. org/10.1177/053901882021004003.

Prahalad, C. K. (2004). The fortune at the bottom of the pyramid. USA: Wharton School Publishing.

Prahalad, C. K., \& Hamel, G. (1990). The core competence of the corporation. Harvard Business Review, 68(3), 79-91.

Premkumar, G., Ramamurthy, K., \& Saunders, C. S. (2005). Information processing view of organizations: an exploratory examination of fit in the context of interorganizational relationships. Journal of Management Information Systems, 22(1), 257-298.

Prietula, M. J., Carley, K., \& Gasser, M. (1998). Simulating organizations: computational models of institutions and groups. Menlo Park: AAAl Press/The MIT Press.

Ree, M. J., Carretta, T. R., \& Steindl, J. R. (2002). Cognitive ability. In D. S. Ones, N. Anderson \& H. K. Sinangil (Eds.), Handbook of industrial, work and organizational psychology: personnel psychology (pp. 219-232). London: Sage.

Reed, S. K. (1988). Cognition: theory and applications (2. ed.). Pacific Grove: Brooks-Cole Publishing Company.

Scherer, K. R. (1982). Emotion as a process: function, origin, and regulation. Social Sciences Information. Information Sur les Sciences Sociales, 21(4-5), 555-570. http://dx.doi. org/10.1177/053901882021004004.

Schmidt, F. L., \& Hunter, J. E. (2000). Select intelligence. In E. A. Locke (Ed.), The blackwell handbook of principles of organizational behavior (pp. 3-14). Oxford: Blackwell Publishers.

Scott, W. R. (1998). Organizations: rational, natural, and open systems. New Jersey: Prentice Hall.

Simon, H. A. (1947). Administrative behavior: a study of decision-making processes in administrative organization. New York: Macmillan.

Simon, H. A. (1982a). Models of bounded rationality: economic analysis and public policy (Vol. 1). Cambridge: MIT Press.
Simon, H. A. (1982b). Models of bounded rationality: behavioral economics and business organization (Vol. 2). Cambridge: MIT Press.

Simon, H. A. (1983). Reason in human affairs. Stanford: Stanford University Press.

Simon, H. A. (1996). The sciences of the artificial (3. ed.). Cambridge: MIT Press.

Simon, H. A. (1997a). Models of bounded rationality: empirically grounded economic reason (Vol. 3). Cambridge: MIT Press.

Simon, H. A. (1997b). Administrative behavior: a study of decision-making processes in administrative organizations (4. ed.). New York: The Free Press.

Spender, J. C. (1996a). Making knowledge the basis of a dynamic theory of the firm. Strategic Management Journal, 17(S2), 45-62. http://dx.doi.org/10.1002/smj.4250171106.

Spender, J. C. (1996b). Organizational knowledge, learning, and memory: three concepts in search of a theory. Journal of Organizational Change Management, 9(1), 63-78. http:// dx.doi.org/10.1108/09534819610156813.

Stehr, N., \& Meja, V. (2005). Society and knowledge: contemporary perspectives in the sociology of knowledge and science (2. ed.). London: Transaction Publishers.

Takahashi, S., \& Takahashi, V. P. (2007). Gestão de inovação de produtos: estratégia, processo, organização e conhecimento. São Paulo: Campus/Elsevier.

Takeuchi, H., \& Nonaka, 1. (2004). Hitotsubashi on knowledge management. Singapore: John Wiley \& Sons.

Venkatraman, N., \& Subramaniam, M. (2002). Theorizing the future of strategy: questions for shaping strategy research in the knowledge economy. In E. T. Pettigrew (Ed.), Handbook of strategy and management (pp. 461-474). Newbury Park: Sage.

Visser, M. (2007). Deutero-learning in organizations: a review and a reformulation. Academy of Management Review, 32(2), 659-667. http://dx.doi.org/10.5465/AMR.2007.24351883.

Von Bertalanffy, L. (1968). General system theory: foundations, development, and applications. New York: Allen Lane.

Walsh, J. P. (1995). Managerial and organizational cognition: notes from a trip down memory lane. Organization Science, 6(3), 280-321. http://dx.doi.org/10.1287/orsc.6.3.280.

Weick, K. E. (1979). The social psychology of organizing (2. ed.). Menlo Park: Addison-Wesley.

Weick, K. E. (1995). Sensemaking in organizations. London: Sage Publications.

Wernerfelt, B. (1984). A resource-based view of the firm. Strategic Management Journal, 5(2), 171-180. http:// dx.doi.org/10.1002/smj.4250050207.

Wiener, N. (1961). Cybernetics or control and communication in the animal and the machine (2. ed.). Cambridge: MIT Press. http://dx.doi.org/10.1037/13140-000.

Zadeh, L. A. (1973). Outline of a new approach to the analysis of complex systems and decision process. IEEE Transactions on Systems, Man, and Cybernetics, 3(1), 28-44. http:// dx.doi.org/10.1109/TSMC.1973.5408575.

Zadeh, L. A. (1999). From computing with numbers to computing with words: from manipulation of measurements to manipulation of perceptions. IEEE Transactions on Circuits and Systems, 45(1), 105-119. http://dx.doi. org/10.1109/81.739259. 


\title{
Organizational cognition: review, conceptualization and strategic context
}

\begin{abstract}
Organizational cognition has benefited from computational and interpretive approaches that have primarily occurred over the last fifty years. This paper recognizes and supports these important developments but argues that organizational cognition must advance toward more concise and sharp conceptualizations with clear distinctions and frontiers regarding organizational intelligence, autonomy, learning, and knowledge management. Additionally, this paper suggests that advancements in organizational cognition must bridge theory and practice. This work reviews existing literature, proposes conceptualizations of organizational cognition, and introduces organizational cognition into the strategic resource context.
\end{abstract}

Keywords

Organizational cognition. Organizational ability. Strategic resources. Environmental uncertainty. 